\title{
Acesso aos serviços de saúde nos municípios do entorno sul do Distrito Federal
}

\section{Access to health services in the southern outskirts of the Brazilian Federal District}

\author{
Ana Cléia Margarida Tonhá ${ }^{1}$, Paulo Roberto Borges de Souza Júnior ${ }^{2}$, \\ Ana Lúcia Queiroz Bezerra ${ }^{3}$, Thays Angélica de Pinho Santos ${ }^{4}$, Claci Fátima Weirich Rosso ${ }^{5}$
}

\footnotetext{
${ }^{1}$ Enfermeira, Mestre em Enfermagem. Enfermeira da Secretaria Municipal de Saúde de Aparecida de Goiânia. Goiânia, GO, Brasil. E-mail: anacleiatonha@gmail.com. ${ }^{2}$ Estatístico, Doutor em Saúde Pública. Pesquisador do Instituto de Comunicação e Informação Científica e Tecnológica da Fundação Oswaldo Cruz. Rio de Janeiro, RJ, Brasil. E-mail: paulo.borges@icict.fiocruz.br.

${ }^{3}$ Enfermeira, Doutora em Enfermagem. Professora Associado da Faculdade de Enfermagem da Universidade Federal de Goiás (FEN/UFG). Goiânia, GO, Brasil. E-mail: analuciaqueiroz@uol.com.br.

${ }^{4}$ Enfermeira, Mestre em Enfermagem. Enfermeira da Secretaria Municipal de Saúde de Aparecida de Goiânia. Goiânia, GO, Brasil. E-mail: thays_angel_8@hotmail.com.

${ }^{5}$ Enfermeira, Doutora em Ciências da Saúde. Professora Adjunto da FEN/UFG. Goiânia, GO, Brasil. E-mail: claci.proec.ufg@gmail.com.
}

\section{RESUMO}

O estudo objetivou analisar a percepção de pessoas residentes sobre o acesso aos serviços de saúde, na região do Entorno Sul da Região Integrada de Desenvolvimento do Distrito Federal e Entorno - RIDE/DF. Pesquisa epidemiológica descritiva, transversal do tipo inquérito domiciliar de base populacional desenvolvida em 2010 e 2011 . Utilizado questionário semiestruturado previamente validado. Foram entrevistados 605 residentes dos municípios do Entorno Sul, desses 311 (51,4\%) são do sexo feminino, 215 (35,8\%) possuem ensino fundamental incompleto, 163 (40,9\%) procuram por atendimento de saúde em Unidades de Saúde e 166 (45,5\%) buscam por atendimento de saúde em outro município. Concluiu-se que o acesso aos serviços de saúde na região é influenciado pela condição social e econômica e pelo local onde as pessoas residem o que permite aos gestores estudarem melhor o cenário, reorientar a forma de atendimento e adequar a assistência à saúde àquela população.

Descritores: Acesso aos Serviços de Saúde; Inquérito de Saúde; Uso de Serviços de Saúde.

\section{ABSTRACT}

The aim of this study was to analyze people's perception on the access to health services in the southern outskirts of the Integrated Region for the Development of the Federal District (RIDE/DF, as per its acronym in Portuguese). A descriptive, cross-sectional, epidemiological and population-based study with a household survey design was carried out in 2010 and 2011. A semi-structured questionnaire, previously validated, was applied. A total of 605 individuals who lived in the southern outskirts of the Federal District were interviewed, of which 311 (51.4\%) were female, 215 (35.8\%) had incomplete primary education, 163 (40.9\%) sought health services in Primary Care Units and 166 (45.5\%) sought health services in another municipality. Access to health services in the region is influenced by social and economic conditions and by the place of living. These findings allow managers to better study the setting, review care services provided and meet health needs of that population.

Descriptors: Health Services Accessibility; Health Surveys; Health Services. 


\section{INTRODUÇÃO}

Desde sua criação em 1988, o Sistema Único de Saúde - SUS deve oferecer a todos os cidadãos o direito à assistência à saúde gratuita direcionada para a promoção, proteção e recuperação da saúde individual e coletiva. As ações devem ser oferecidas desde Atenção Básica à Atenção de Alta Complexidade garantindo, dessa forma, o acesso integral a toda população brasileira aos serviços de saúde em qualquer nível de complexidade ${ }^{(1)}$.

Ao longo do processo da implantação do SUS, a saúde é tida como produção social, ou seja, tem implicações determinadas por uma rede de causas e efeitos que envolvem elementos sociais, econômicos e culturais que se processam e se sintetizam na experiência individual, de cada grupo e da sociedade ${ }^{(2)}$.

Desde a implantação do SUS, o acesso aos serviços de saúde no Brasil tem aumentado consideravelmente. De acordo com a Pesquisa Nacional por Amostra de Domicílios - PNAD realizada em 1981, antes da criação do SUS, $8 \%$ de toda população brasileira afirmava ter usado algum tipo de serviço de saúde pública, enquanto em $2008,14,2 \%$ da população relatava uso de algum tipo de serviço de saúde pública ${ }^{(3)}$.

Apesar do aumento do uso dos serviços de saúde públicos no Brasil, ainda é necessário avançar muito para assegurar os mínimos padrões de assistência de qualidade. Nesse contexto, o SUS enfrenta amplas dificuldades relacionadas à estruturação do novo modelo de atenção à saúde, assim como do acesso da população a esses serviços, comprometendo o seu desempenho.

Nesse sentido essas dificuldades comprometem a resolutividade dos serviços de saúde, já que, na proposta de consolidação do SUS, existe uma regulamentação para operacionalizar os serviços prestados. Ao mesmo tempo, não há efetivação do processo de descentralização ao nível mais operacional. Sinalizando haver uma incoerência entre os propósitos de descentralização das ações de saúde e a forma de organização dos serviços na rede básica de saúde ${ }^{(4-5)}$.
O direito ao acesso a todos os tipos de serviços de saúde pela população brasileira está diretamente relacionado com a aplicação cotidiana dos princípios organizativos e doutrinários do SUS, a partir da corresponsabilidade de cada esfera governamental, trabalho conjunto dos gestores, dos trabalhadores que prestam serviços ao SUS e dos usuários ${ }^{(2)}$.

$\mathrm{Na}$ corresponsabilidade assumida pelo ente federado, tem se destacado o processo de descentralização e regionalização, por meio de redes e de conexões que se formam para organizar os serviços de saúde em suas muitas complexidades, como a Atenção Primaria à Saúde (APS), em especial com a Estratégia Saúde da Família (ESF) que traz em sua concepção um novo modelo assistencial em que o vínculo com a população adstrita é privilegiado. A ESF é um modelo assistencial que tem como lógica atender ao cidadão, à família e à comunidade de maneira integral e ao direito de ser cuidado por uma equipe de saúde de maneira resolutiva $^{(6)}$.

O acesso à saúde tem sido o objeto de muito interesse dos gestores públicos, entretanto o conceito de acesso à saúde tornou-se mais complexo. As primeiras análises, datadas da década de 1970, sugeriam uma forte relação do acesso com o aspecto geográfico (disponibilidade) e financeiro (capacidade de pagamento). A literatura mais recente $e^{(6-7)}$ procura abordar aspectos menos tangíveis, como os aspectos cultural, educacional e socioeconômico, incorporando o elemento aceitabilidade nas análises. A literatura mostra ainda que ter acesso à informação está associado aos conceitos de empoderamento e de letramento tanto dos usuários quanto dos gestores dos serviços de saúde, para a tomada de decisões ${ }^{(7)}$.

Estudiosos $^{(7)}$ associam a definição de acesso a quatro elementos principais, denominados disponibilidade, aceitabilidade, capacidade de pagamento e informação que cada vez mais se confundem com o conceito de equidade em saúde. 
Segundo estudo ${ }^{(8)}$, o acesso aos serviços de saúde tem sido objeto de análise na literatura internacional, acirrando a existência de barreiras aos usuários, como é o caso das filas para marcação de consulta e atendimento e da escassez de estratégias para sua superação. Afirmam ainda que as desigualdades de acesso encontram-se como um dos principais problemas a serem enfrentados, para que o SUS funcione efetivamente, conforme as diretrizes e os princípios propostos.

Em análise do contexto internacional, estudiosos ${ }^{(9)}$ apontam uma necessidade de desenvolver estudos rigorosos para avaliar os efeitos sociais do acesso e da qualidade dos serviços de saúde em países de baixa e média renda.

De modo geral, as pesquisas sobre acesso e utilização de serviços de saúde são focalizadas na demanda presente nos serviços, nas características demográficas e nos problemas de saúde com maior prevalência. Esse tipo de pesquisa, portanto exclui pessoas que não procuram esses serviços e comprometem o real conhecimento em nível populacional ${ }^{(6)}$. Nesse sentido, é importante analisar como as pessoas residentes nos municípios no entorno no Distrito Federal percebem o acesso ao atendimento nos serviços de saúde e fatores que impedem o seu acesso. Dessa forma será possível subsidiar a tomada de decisão dos gestores locais e estaduais para assim, ampliar a porta de entrada aos usuários na Atenção Primária.

O presente estudo teve como objetivo analisar a percepção de pessoas residentes sobre o acesso aos serviços de saúde, na região do Entorno Sul da Região Integrada de Desenvolvimento do Distrito Federal e Entorno - RIDE/DF.

\section{MÉTODOS}

Estudo epidemiológico descritivo e transversal do tipo inquérito domiciliar de base populacional, parte de um inquérito domiciliar da Região Integrada de Desenvolvimento do Distrito Federal e Entorno RIDE/DF, composto por municípios do Entorno Sul. Tratase de uma etapa de projeto desenvolvido pela
Universidade Federal de Goiás - UFG e a Universidade de Brasília - UnB, em parceria com a Fundação Osvaldo Cruz - FIOCRUZ, intitulado "Apoio à Regionalização nos Municípios da RIDE-DF". Os municípios que tiveram setores censitários selecionados para o estudo foram: Santo Antônio do Descoberto, Águas Lindas, Novo Gama, Valparaíso, Luziânia e Cristalina.

A amostra do estudo foi constituída por pessoas maiores de 18 anos de idade, residentes nos domicílios selecionados aleatoriamente nos municípios. Foram excluídas aquelas que apresentaram evidência de transtorno mental grave ou qualquer condição que as incapacitasse de responder ao questionário no momento da abordagem, mediante diagnóstico médico.

O tamanho da amostra foi calculado para detectar diferenças de até 3,5\% em uma proporção de 15\%, com um poder de teste de $80 \%$ e um nível de significância de 95\%. Por se tratar de um desenho complexo de amostragem, incorporou-se um efeito de desenho de 1,3, pois foi necessário entrevistar 2.400 indivíduos no total, sendo 600 indivíduos em cada estrato - região da RIDE/DF. Levando-se em consideração possíveis perdas, em torno de $20 \%$, aumentou-se o tamanho da amostra em cada estrato - de 600 para 720 domicílios, totalizando 2.880 domicílios nos quatro estratos. Esse tamanho da amostra levou em conta as quatro regiões do entorno: Região Sul, Região Norte, Região dos Pirineus e Região Unaí. Ao término da coleta de dados, foram obtidas 605 entrevistas no estrato do Entorno Sul.

A coleta de dados foi realizada no período de outubro de 2010 a abril de 2011, utilizando-se um instrumento adaptado da Pesquisa Mundial de Saúde - Atenção Básica (PMS-AB), já validada por pesquisadores do ICICT/FIOCRUZ ${ }^{(10)}$. O instrumento é composto por duas partes: questionário domiciliar e questionário individual e foi ajustado após discussões com as equipes da UFG, UnB e FIOCRUZ. O questionário domiciliar contém perguntas sobre as características do domicílio, características socioeconômicas e de saúde dos residentes, assim como cobertura, acesso e utilização da Estratégia de Saúde da 
Família. O questionário individual está dividido em dez módulos nomeados de "A a J" com questões relacionadas a diversos temas. $O$ estudo utilizou ambos os questionários do instrumento. Do questionário domiciliar utilizaram-se as variáveis relacionadas à saúde dos residentes do domicílio, como também cobertura, acesso e utilização da Estratégia de Saúde da Família; do questionário individual utilizou-se o Módulo $A$ (características sociodemográficas e apoio social) e Módulo J (Desempenho do Sistema de Saúde e acesso e utilização dos serviços de saúde).

Os domicílios foram selecionados pelos supervisores de campo mediante uso de mapas dos municípios com as demarcações dos setores censitários, que são conjuntos de domicílios utilizados pelo Instituto Brasileiro de Geografia e Estatística - IBGE para coleta do Censo Demográfico de $2010^{(11)}$. Juntamente com os mapas foram fornecidas Fichas de Identificação do Setor - FIS, com as demarcações dos setores censitários, juntamente com o valor do "pulo"- intervalo de amostragem que é a quantidade de domicílios que deverá existir entre um domicílio selecionado e outro. Esses valores de "pulo" variam de acordo com o tamanho do setor censitário, de modo a contemplar domicílios selecionados de toda a extensão do setor delimitado.

Ao morador selecionado presente no domicílio, foram apresentados os objetivos do estudo e solicitada a assinatura do Termo de Consentimento Livre e Esclarecido para participar do estudo.

Os dados foram digitados em formulário eletrônico na internet, acessíveis apenas, por meio de senha pessoal. O formulário eletrônico foi desenvolvido pela ICICT FIOCRUZ.

A análise estatística foi realizada com o auxílio do pacote estatístico Statistical Package for Social Science versão 20.0. As variáveis foram apresentadas no formato de razões e proporções.

O projeto foi submetido e aprovado pelo Comitê de Ética e Pesquisa da Faculdade de Saúde da Universidade de Brasília (FR 342534) e contou com o apoio do
Colegiado de Gestão da Saúde da RIDE - DF, seguindo as recomendações da legislação brasileira para condução de projetos de investigação com seres humanos do Conselho Nacional de Saúde.

\section{RESULTADOS}

Participaram desse estudo 605 pessoas residentes em áreas urbanas e rurais dos municípios do Entorno Sul do DF, selecionadas de forma aleatória, sistemática e segundo os critérios metodológicos dessa pesquisa. Os resultados referentes à caracterização dos residentes e ao acesso aos serviços de saúde estão evidenciados abaixo.

\section{Caracterização sociodemográfica e econômica dos residentes do Entorno Sul da RIDE-DF}

Dos 605 entrevistados, 311 (51,4\%) são do sexo feminino e situam-se na faixa etária entre 25 a 39 anos, 255 (42,2\%), de um grupo relativamente jovem. Quanto ao Grau de Instrução, 141 (23,5\%) possuem Ensino Fundamental completo, 405 (67,3\%) são casados ou vivem com o companheiro, 309 (51,1\%) dos residentes se autorreferem da cor parda, 368 (60,9\%) estavam empregados no momento da entrevista, 255 (42,1\%) tem renda de um a três salários mínimos e 144 (23,8\%) possuem renda mensal abaixo de um salário mínimo conforme Tabela 1.

\section{Acesso aos serviços de saúde}

Em relação ao acesso aos serviços de saúde dos residentes dos municípios do Entorno Sul, foram analisadas somente as respostas dos residentes que passaram por algum tipo de atendimento em saúde nos últimos 12 meses. Os resultados estão apresentados nas Tabelas 2 e 3. 
Tabela 1: Perfil sociodemográfico dos residentes do Entorno Sul, RIDE-DF. Distrito Federal, Brasil, 2012.

\begin{tabular}{|c|c|c|}
\hline Variáveis & Frequência $(n=605)$ & $\%$ \\
\hline \multicolumn{3}{|l|}{ Sexo } \\
\hline Feminino & 311 & 51,4 \\
\hline Masculino & 294 & 48,6 \\
\hline \multicolumn{3}{|l|}{ Faixa Etária (anos) } \\
\hline 18 a 24 & 111 & 18,4 \\
\hline 25 a 39 & 255 & 42,2 \\
\hline 40 a 59 & 191 & 31,5 \\
\hline 60 e mais & 48 & 7,9 \\
\hline \multicolumn{3}{|l|}{ Grau de instrução } \\
\hline Menos de 1 ano de estudo & 59 & 9,9 \\
\hline Fundamental incompleto & 215 & 35,8 \\
\hline Fundamental Completo & 141 & 23,5 \\
\hline Ensino Médio Completo & 186 & 30,8 \\
\hline \multicolumn{3}{|l|}{ Estado Civil } \\
\hline Solteiro & 135 & 22,4 \\
\hline Casado ou vive com companheiro (a) & 405 & 67,3 \\
\hline Divorciado ou separado & 39 & 6,4 \\
\hline Viúvo & 23 & 3,8 \\
\hline Não souberam responder & 5 & 0,8 \\
\hline \multicolumn{3}{|l|}{ Cor da pele (autorreferida) } \\
\hline Branca & 174 & 28,8 \\
\hline Preta & 80 & 13,2 \\
\hline Parda & 309 & 51,1 \\
\hline Outra & 42 & 6,9 \\
\hline \multicolumn{3}{|l|}{ Situação de trabalho } \\
\hline Trabalha atualmente & 368 & 60,9 \\
\hline Não trabalha atualmente & 237 & 39,1 \\
\hline \multicolumn{3}{|l|}{ Renda } \\
\hline Não tem rendimento & 91 & 15,0 \\
\hline Até um salário mínimo & 144 & 23,8 \\
\hline De um a três salários mínimos & 255 & 42,1 \\
\hline Acima de três salários mínimos & 78 & 12,9 \\
\hline Recusou a responder & 37 & 6,2 \\
\hline
\end{tabular}

Tabela 2: Tipos de estabelecimentos de saúde mais procurados pelos moradores do Entorno Sul, RIDE-DF. Distrito Federal, Brasil, 2012.

\begin{tabular}{ccc}
\hline Estabelecimentos & Frequência $(\mathbf{n = 3 9 9 )}$ & \% \\
\hline Unidade de saúde pública & 163 & 40,9 \\
Unidade de Pronto Atendimento (UPA) & 7 & 1,8 \\
Outro tipo de Pronto & 13 & 3,3 \\
Pronto-socorro ou Emergência de hospital público & 73 & 18,3 \\
Ambulatório de hospital público & 55 & 13,8 \\
Consultório particular & 70 & 17,5 \\
Pronto-socorro ou emergência de hospital privado & 17 & 4,3 \\
Outro & 1 & 0,3 \\
\hline
\end{tabular}


Tabela 3: Local do serviço, tempo de deslocamento, meio de transporte, procedimento para conseguir atendimento e atendimento feito pelo SUS aos residentes do Entorno Sul. Distrito Federal, Brasil, 2012.

\begin{tabular}{lcc}
\hline \multicolumn{1}{c}{ Variáveis } & Frequência (n=365) & \% \\
\hline Local do serviço de saúde & 196 & 53,7 \\
$\quad$ No mesmo município que o residente mora & 166 & 45,5 \\
Em outro município & & \\
Tempo de deslocamento & 337 & 92,3 \\
$0-1$ hora & 28 & 7,7 \\
$\quad$ horas e mais & & \\
Meio de transporte utilizado & 131 & 35,9 \\
Transporte coletivo & 142 & 38,9 \\
Carro ou motocicleta & 5 & 1,4 \\
Ambulância & 74 & 20,3 \\
A pé & 11 & 3,0 \\
Bicicleta & & \\
Procedimento para conseguir atendimento & 206 & 56,4 \\
Foi direto ao serviço de saúde, sem marcar consulta & 149 & 40,8 \\
Agendou a consulta previamente & & \\
Atendimento realizado ou não pelo SUS & 255 & 69,9 \\
Atendimentos realizados pelo SUS & 109 & 29,9 \\
$\quad$ Atendimentos não realizados pelo SUS & & \\
\hline
\end{tabular}

Quanto ao tipo de estabelecimento de saúde procurado pelos residentes, 163 (40,9\%) buscaram atendimento em Unidades de saúde pública, 73 (18,3\%), em Pronto-socorro ou emergência de hospital público e 70 (17,5\%), em Consultório particular.

A Tabela 3 mostra que 166 (45,5\%) se deslocaram para atendimento em saúde em outro município, sendo o meio de "transporte coletivo" o mais utilizado por 131 $(35,9 \%)$ residentes.

Dos residentes que procuraram por atendimento nos serviços de saúde, 206 (56,4\%) foram direto ao serviço de saúde sem marcar nenhum tipo de consulta.

Quanto ao convênio do atendimento recebido, 255 (69,9\%) foram pelo SUS e 109 (29,9\%), por outros convênios.

\section{DISCUSSÃO}

O perfil dessa região é de pessoas do sexo feminino, apontando uma maior demanda de saúde da mulher e da criança nos serviços de saúde. O que reforça tal caracterização na região estudada é o fato de que, ao serem indagadas sobre o motivo de estarem desempregadas no momento da entrevista, a resposta é a atividade "do lar", que reforça o papel que a mulher assume como cuidadora do lar, de si e da própria família $^{(12)}$.

De modo geral, as pessoas da região como adultos jovens, cujo grau de instrução é bastante diversificado, sendo que a maior parte possui Ensino Fundamental Incompleto e destaca-se que 59 (9,9\%) dos residentes têm menos de um ano de estudo.

Uma das características mais marcantes da população das localidades com maiores precariedades é a baixa escolaridade, assim como dos participantes do estudo. Tal situação, possivelmente, desenha o quadro de fragilidade desses residentes e de falta de autonomia para mudar a realidade local. O que, consequentemente, torna tais municípios vulneráveis às condições locais, porque não têm aí a possibilidade de desenvolver seu potencial. O que leva à maior dificuldade de inserção no mercado de trabalho e, também, de ascensão dos menos qualificados $^{(13)}$.

Ainda considerando o grau de instrução, a literatura mostra que é possível associá-lo ao acesso aos serviços de 
saúde ${ }^{(8)}$. Uma interpretação possível é a de que ter acesso à informação está na base do acesso à saúde, estando essa noção associada aos conceitos de empoderamento do usuário para tomada de decisões no seu processo terapêutico.

Quanto à cor autorreferida, 309 afirmaram ser da cor parda $(51,1 \%)$. Esses resultados reproduzem a realidade nacional de acordo com a pesquisa do IBGE sobre as características étnico-raciais da população - PCERP realizada em 2011. Na referida pesquisa, os entrevistados citaram algumas circunstâncias em que a cor tem influenciado a vida das pessoas no Brasil tais como: no trabalho, na relação com a justiça/polícia, no convívio social, além de influenciar em escolas e repartições públicas $^{(14)}$.

Uma interpretação possível é que há anos o Brasil tem sofrido um intenso processo de miscigenação. Não diferente, os municípios do Entorno Sul, em seu processo de formação histórica junto à Capital Federal, abrigam inúmeros migrantes de diferentes estados do Brasil, atraídos à nova capital por perspectivas de melhores condições de vida e oportunidade de trabalho ${ }^{(15)}$. Tal situação, além de refletir a condição de miscigenação típica do Brasil, ainda, evidencia o grande fluxo de migração pendular no país, em especial no Entorno da Capital Federal.

Apesar da maioria dos residentes estar empregada de forma remunerada, no momento da entrevista, uma parcela das pessoas residentes se encontrava economicamente inativa, ou seja, desempregada, aposentada ou exercendo trabalho não remunerado. A falta de empregos e equipamentos urbanos nas cidades que compõem a região do Entorno do Distrito Federal geram um movimento pendular cada vez maior em direção à Capital Federal.

Essa população desassistida busca por empregos, serviços hospitalares e educação na Capital Brasileira, evidenciando características de "cidades dormitório", em que os residentes trabalham durante o dia fora de sua cidade de origem e retornam durante a noite. A falta de equipamentos urbanos, também, inclui a precariedade dos serviços de saúde. Alguns municípios do Entorno Sul do DF, região do estudo, são considerados área metropolitana de Brasília, e por isso, exercem relações comerciais fortes com a Capital Federal fortalecendo ainda mais essa dependência com o DF ${ }^{(15)}$.

Boa parte dos residentes do estudo possui renda inferior a um salário mínimo, o que possivelmente pode dificultar o acesso a serviços de saúde, acesso à escolaridade, à capacitação profissional, a empregos. Fatores esses que estão associados ao alto índice de violência urbana e criminalidade características dessa região do Entorno.

Dados da Pesquisa Nacional por Amostra de Domicílios - PNAD ${ }^{(16)}$ apontam que a renda influenciou mais o acesso do que a escolaridade. Em particular, a renda mostrou-se fator importante no acesso aos serviços de saúde das crianças. Apesar da permanência de desigualdades sociais, no acesso aos serviços de saúde, houve uma tendência de redução de sua magnitude no país como um todo ${ }^{(17)}$.

Ainda, conforme resultados da PNAD analisados por alguns autores ${ }^{(16-17)}$, esse estudo reafirma, também, outro padrão: que o acesso aos serviços de saúde na região de estudo é claramente influenciado pela condição social das pessoas e pelo local onde residem. Desigualdades no acesso nem sempre são observadas em outros países e sua presença ou não expressam as especificidades do sistema de saúde de cada região.

Os residentes relataram levar até uma hora para chegar ao serviço de saúde. Percebe-se ainda, nessa parcela, que o meio de transporte mais citado foi "carro ou moto", seguido de "transporte público". Somando esse tempo à jornada de trabalho diária, o indivíduo fica supostamente sem tempo para incorporar à sua rotina diária aspectos de uma vida mais saudável para si próprio e para sua família, como atividade física, atividades de lazer por exemplo.

Para uma melhor abordagem dessa problemática, seria necessário saber a localização do serviço de saúde 
que as pessoas residentes buscam quando necessitam de cuidados em saúde.

Essa informação possibilitaria a compreensão da lógica de procura dos serviços realizada pelos residentes, a identificação de problemas assistenciais, o levantamento de movimentos migratórios pendulares. $\mathrm{O}$ que serviria para a utilização de serviços de saúde ou, ainda, a verificação posterior junto à gestão local da existência de transporte público efetivo para se acessar esses serviços ${ }^{(18-19)}$.

No que se refere à saúde, é possível perceber que os moradores dessa região por vezes se veem desassistidos em suas localidades, já que os serviços de saúde se encontram, muitas vezes, impossibilitados de fornecer atendimento de saúde digno.

Embora pertençam ao Estado de Goiás, esses municípios se encontram geograficamente distantes da capital Goiânia e sua região metropolitana. Por isso, acabam por ter como alternativa, buscar atendimento médico no Distrito Federal, inflando os serviços. Por se tratar, também, de cidades consideradas “cidades dormitório", a população permanece no período diurno nos locais de trabalho no DF e ao retornar para seus municípios à noite, encontrar a maioria dos serviços de saúde fechada, tendo dessa forma poucas alternativas de busca por serviços de saúde.

\section{CONCLUSÃO}

O acesso aos serviços de saúde da região de estudo é claramente influenciado pela condição social das pessoas e pelo local onde residem.

O Sistema Único de Saúde, apesar de representar um enorme avanço em termos de políticas públicas de saúde no Brasil, ainda não foi capaz de fazer face às desigualdades no acesso aos serviços de saúde, em especial em regiões que apresentam peculiaridades locais.

Conhecer as barreiras e aumentar a oferta de serviços com perfil de funcionamento adequado para quem trabalha, é fundamental para tentar reduzir as iniquidades observadas. Por outro lado, promover a inserção no trabalho com garantias dos direitos sociais pode contribuir para a melhoria da saúde pública. Assim, a melhora da qualidade de acesso e utilização dos serviços de saúde dependem entre outros fatores, principalmente, do uso de políticas públicas integradas e efetivas.

20];14(34): 633-45. Disponível em:

http://www.scielo.br/scielo.php?script=sci arttext\&pid=S141432832010000300013.

6. Tesser CD, Norman AH. Repensando o acesso ao cuidado na Estratégia Saúde da Família Saude Soc [ Internet]. 2014 Set [Acesso em: 2015 Fev 20] ; 23( 3 ): 869-883. Disponível em:

http://dx.doi.org/10.1590/S0104-12902014000300011.

7. Sanchez RM, Ciconelli RM. Conceitos de acesso à saúde. Rev Panam Salud Publica. [Internet]. 2012 [Acesso em: 11 abr 2014]; 31(3): 260-8. Disponível em: http://www.scielosp.org/pdf/rpsp/v31n3/12.pdf. 8. Assis MMA, Jesus WLA. Acesso aos serviços de saúde: abordagens, conceitos, políticas e modelo de análise. Cien Saude Colet [Intenet]. 2012 [Acesso em: 11 abr 2014]; 17(11): 2865-75. Disponível em: http://www.scielo.br/pdf/csc/v17n11/v17n11a02.pdf.

9. Lagarde M, Palmer N. The impact of user fees on access to health services in low-and middle-income countries. Cochrane Database of Systematic Reviews [Internet]. 2011 [Acesso em:11 abr 2014]; 4: 1-68. Disponível em: http://apps.who.int/rhl/reviews/CD009094.pdf. 10. Swarcwald CL, Viacava F. Pesquisa Mundial de Saúde: Aspectos metodológicos e articulação com a Organização Mundial de Saúde. Rev. Bras. Epidemiol [Internet]. 2008 [ Acesso em 11 abr 2014]; 
11(suppl 1): 58-66. Disponível em:

http://www.scielo.br/pdf/rbepid/v11s1/05.pdf.

11. IBGE- Instituto Brasileiro de Geografia e Estatística. Pesquisa

Nacional por Amostra de Domicílios, 2011. Brasília: IBGE [Internet].

2011 [Acesso em: 11 abr 2014]. Disponível em:

http://www.ibge.gov.br/home/estatistica/pesquisa_resultados.php?id.

12. Pereira RA, Santos EB, Fhon JRS, Marques S, Rodrigues RAP.

Sobrecarga dos cuidadores de idosos com acidente vascular cerebral.

Rev Esc Enferm USP [Internet]. 2013 Fev [Acesso em 23 fev 2015];

47(1): 185-92. Disponível em:

http://www.scielo.br/pdf/reeusp/v47n1/a23v47n1.

13. Carinhanha Joana labrudi, Penna Lucia Helena Garcia. Violência

vivenciada pelas adolescentes acolhidas em instituição de

abrigamento. Texto contexto - enferm. [Internet]. 2012 Mar [Acesso

em 24 fev 2015] ; 21(1): 68-76. Disponível em:

http://dx.doi.org/10.1590/S0104-07072012000100008.

14. Chor D, Desigualdades em Saúde no Brasil: É preciso ter raça. Cad.

Saúde Pub. Rio de Janeiro, 2013;29(7):1272-1275.

15. Caiado MCS, Estruturação intra-urbana na região do Distrito Federal e entorno: a mobilidade e a segregação socioespacial da população. Rev. Bras. Est. Pop. São Paulo, 2013: 22(1): 55-58.

16. IBGE - Instituto Brasileiro de Geografia e Estatística [Internet].

Brasília: Ministério do Planejamento, Orçamento e Gestão. Pesquisa

Nacional por Amostra de Domicílios. Síntese de Indicadores 2005.

Brasília, IBGE [Internet]. 2005 [Acesso em 11 abr 2014] Disponível em:

http://www.ibge.gov.br/home/estatistica/populacao/trabalhoerendim ento/pnad2005/default.shtm.

17. Nunes B, Thume E, Tomasi E, Duro SMS, Facchini LA. Desigualdades socioeconômicas no acesso e qualidade da atenção nos serviços de saúde. Rev. Saúde Pub. São Paulo, 2014: 48(6) 968-976.

18. Borim FSA, Neri AL, Francisco PMSB, Barros MBA. Dimensions of self-rated health in older adults. Rev. Saúde Pub. São Paulo, 2014:

48(5): 714-722.

19. Giatti L, Barreto SM. Situação no mercado de trabalho e utilização de serviços de saúde no Brasil. Cien Saude Colet [Internet]. 2011

[Acesso em: 11 abr 2014]; 16(9): 3817-27. Disponível em:

http://www.scielo.br/scielo.php?pid=S1413-

$\underline{81232011001000017 \& \text { script=sci_arttext. }}$.

Recebido: 11/04/2014.

Aceito: 03/02/2015.

Publicado: 30/06/2015. 